\title{
All We Are Saying Is Give Plants a Chance!
}

\author{
Peter M. Gresshoff* \\ Department of Botany, The University of Queensland, Qld 4072, Australia
}

Plant functional genomics have come of age, not only because rice and the model Arabidopsis thaliana are fully DNA sequenced and hundreds of thousands of expressed sequence tags (ESTs) are available on data bases, but because of the isolation of genes defined by mutations controlling plantmicrobe interactions, flowering, plant architecture, and hormone perception. Through this, we learnt that many processes in plants actually share common genetic determinants with bacteria and animals. Yet, public perception and government funding favor biomedical research in the myopic pursuit of our species' seemingly central position in the universe.

There is much excitement as, concurrent with the development in the human genome projects, computing, and robotics and related high throughput technologies, plant biotechnology in the last ten years has made significant advances that will benefit the life on this planet. Sadly, there is no Nobel Prize for plant science, preventing the popular press from featuring achievements in this area $(\mathrm{NB}$, since the inception of Nobel Prizes one century ago, only three were awarded for plant-related research).

We all accept that plants are important in economical, environmental, and ecological terms. Imagine a world without flowers, shade trees, wooden furniture or floors, riverbanks without trees, mountains without meadows, bakeries without bread, glasses without wine, and grocery shops without fruits and vegetables. Oh, I almost forgot, air without oxygen.

Plants also tell us about biology. Most plant cells are pluripotent, indeed totipotent, and the growth of the soma occurs from clusters of stem cells called meristems, from which all organs and cell types derive. Mendel discovered the basic laws of genetics not with bees but peas. The first cloning from a differentiated somatic cell involved not Dolly but tobacco [NB, Dolly and all others like her are not really clones as the mitochondrial DNA is still derived from the egg donor; they are nuclear transplants]. The first recognized gaseous hormone was not nitrous oxide but ethylene. The first discovered morphogen was not retinoic acid but cytokinin. Catalytic RNA was first suggested in viroids attacking avocado. Transposable elements were discovered in corn before bacteria, yeast, and fruit fly. Plant development offers an alternative paradigm for development as most organs are induced postembryonically, relying on the precise perception of chemical signals.

Despite the different life strategies of plants, genomics studies show that many genes and gene systems are in common. For example, a cascade similar to that used in insulin reception perceives the plant hormone indole acetic acid. The hormone, ethylene, is perceived by a two component histidine protein kinase, similar to that found in environmental sensors in bacteria. The same receptor also interacts with nitrous oxide (NO), making one wonder what ethylene can do in humans. Abscissic acid, known in plants to control senescence, was found in human brains. When plants are injured by insects, they release gaseous jasmonic acid that prepares other plant tissues, including those of other species, to resist the attack by inducing protease inhibitors. For example, when you cut your lawn, the roses respond! Microbes that attack plants communicate with their host by secreting a peptide avirulence factor, that suppresses the immune response of the plant. Plants lack immunoglobulintype antibodies but generate diversity through chemically complex mixtures of tannins, flavones, and isoflavones, capable of large combinatorial substitutions. Many of these flavonic compounds are clinically significant as antioxidants, phytoestrogens, and tumor-suppressing compounds. Other secondary plant metabolites such as sucrose, (inchocolate) ethyl-benzylamine, caffeine, nicotine, tetra-hydrocannibinol, theophylline, atropin, aspirin, and warfarin contribute to our life whether legally or not.

Recent gene discoveries in plants have focused on a set of receptor-like protein kinases. These proteins have the ability to interact with other proteins and peptides, opening the possibility that extracellular peptides are critical in plant developmental responses. They also demonstrate that similar control points exist for signal transduction cascades, involving MAP kinases and GTP-binding proteins, commonly found in animal cells. For example, the regulation of root nodule formation in legumes is systemic, as the root communicates to the leaf after infection, and the leaf "replies" to the developing primordium. This process is disrupted in mutants of a receptor-like kinase and leads to supernodulation. Coupled with the recent discovery that post-transcriptional 
gene silencing in plants involves short RNA molecules and that these are capable of long distance movement, we now have to accept that plants possess sophisticated sensing and communication akin to that of animals.

In our pursuit of knowledge, we are frequently awed the efforts in biomedicine and engineering technologies. Indeed, as a species, we are rather selfish in our self-interest. So often I do see gifted undergraduate students, focused on becoming biomedical researchers, recognizing with bewilderment that molecular biology, genomics, and developmental biology also exist in plants! How often does a family member allocate part of an estate to plant research? For interest, notice the average research grant given by NIH compared to USDA.

One of the major breakthroughs came in the mid-1980s, when it became routinely possible to transfer genes into plants, such as the model tobacco (Nicotiana tabacum) and Arabidopsis thaliana. The former had great utility because of its ability to regenerate from totipotent cells. However, tobacco has poor genetics and a rather large and tetraploid genome, so more progress was achieved with the small seeded diploid crucifer Arabidopsis thaliana. Arabidopsis, while relatively poor as a subject for biochemical and chemical analyses because of its size, features a small genome, short generation time, the ability to be transformed efficiently, and an abundance of developmental mutants. These features attracted numerous researchers in other plant species as well as human biology, fungal and bacterial genetics, and Drosophila to culminate in the Arabidopsis Genome Initiative that just months ago released the first complete genomic sequence of the plant. The sequence of about $116 \mathrm{Mbp}$ (representing 90\% of the whole genome as centromeric and telomeric repeat regions remain to be sequenced and aligned) revealed several important features. For one, many gene families and duplications exist, indeed, many are conserved across the eukaryotes. Over 100 Arabidopsis genes are similar to those involved in human hereditary disorders. Also, the plant possesses about the same number of genes as an animal (some 25,000). Progressively, it becomes clear that evolution uses pre-existing genes or gene segments in rearranged, amplified, and mutated ways for alternative applications. Almost a "Virchow Principle of Genes." Perhaps the Buddhist religion has long recognized these holistic connections, hidden from us in the Judeo-Christian-Islamic world, placing our species clearly closest to the deity.

Arabidopsis can be transformed by floral dip as well as seed imbibition. It is possible to merely dip developing flowers into a broth of the natural pathogen, Agrobacterium tumefaciens, harboring plasmid borne genes, and then to select resultant seeds for the expression of the dominant transgene. The elucidation of the entire Agrobacterium-based gene transfer system truly represents a major intellectual achievement (yet no Nobel Prize for it!). Here is a prokaryote that naturally transfers part of a plasmid via a type three like secretion system to the eukaryotic plant host, where the bacterial genes are integrated and expressed to cause neoplasmic transformation. This process of interkingdom gene transfer, which is now well understood, provides the basis for much of agricultural genetic engineering. Roundup Ready soybean and insect-resistant potato all relied on Agrobacterium in their phylogeny.

The genomic analysis was matched by a functional genomic approach, not only in Arabidopsis but also in model species such as the legume Lotus japonicus and Medicago truncatula, as well as crop species such as corn (Zea mays), rice or (Oryza sativa), and soybean (Glycine max). Important gene regulatory networks are being discovered in plants through functional genomic analysis using gene expression analysis, knockout mutations, proteomics coupled with metabolomics, cell ablation, computational modeling, promoter gene fusions, and transgene analysis in multigene combinations.

With the discoveries of regulatory and sensing genes, the potential to alter plant architecture and metabolism, one can be assured that plant biotechnology will impact our lives by transferring paradigms from Arabidopsis and the model legumes to crops. Hopefully, the improved quality of plants around us will aid biomedicine and our species by buying time, so that, we can solve the real social and political problems at hand.

Peter M. Gresshoff

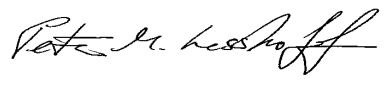

* E-mail: p.gresshoff@botany .uq.edu . au 

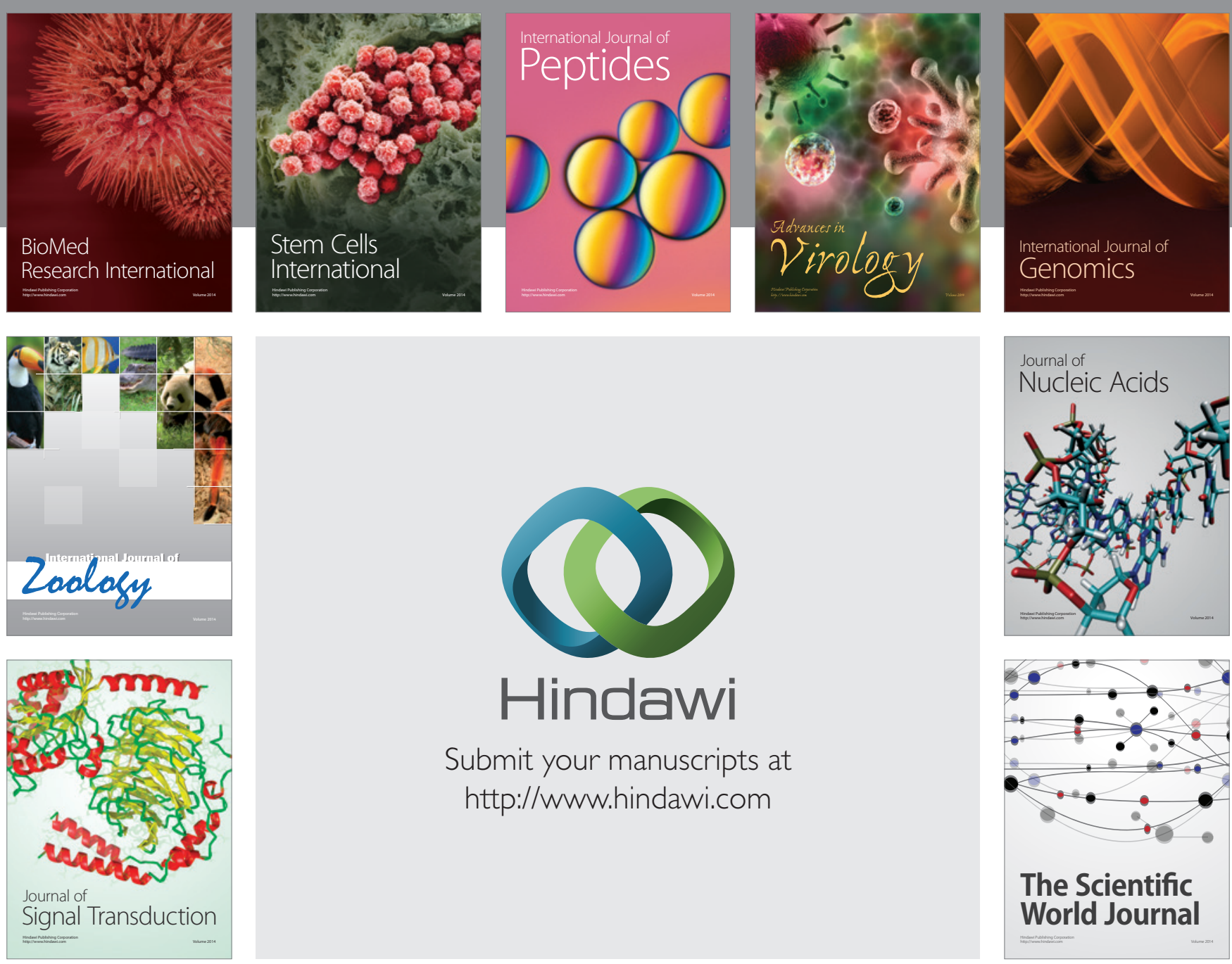

Submit your manuscripts at

http://www.hindawi.com
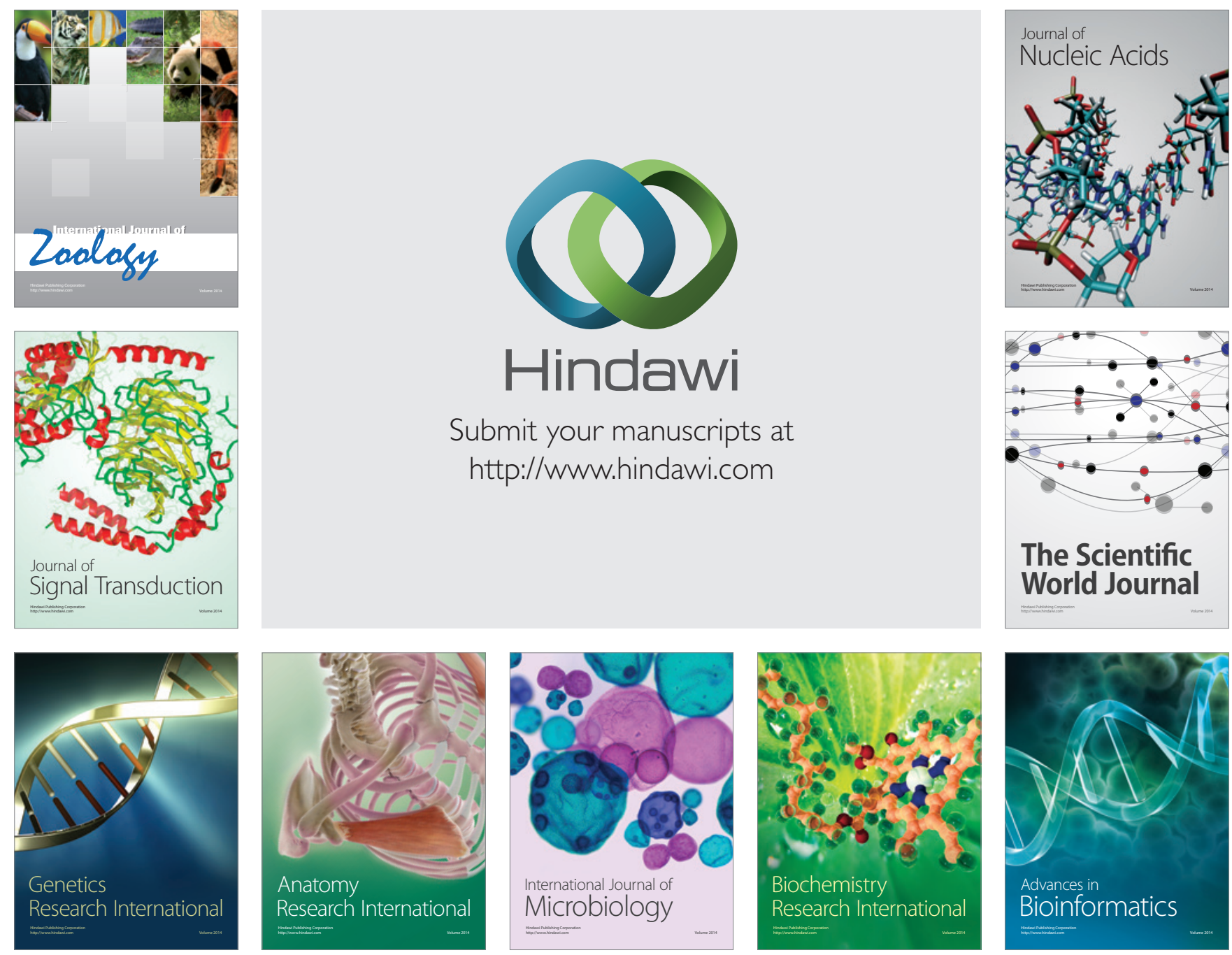

The Scientific World Journal
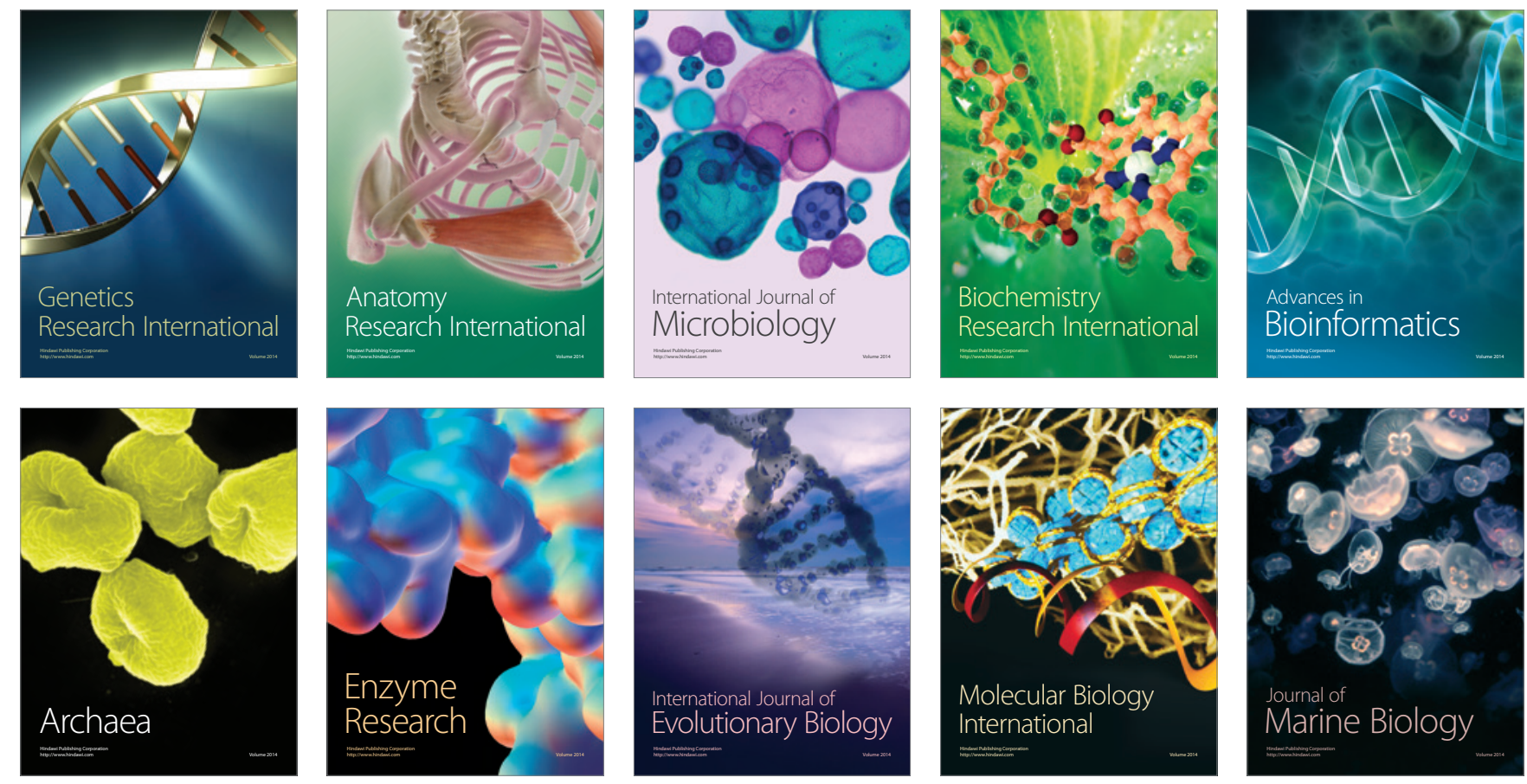